() OPEN ACCESS

\title{
Handheld chromatic pupillometry can accurately and rapidly reveal functional loss in glaucoma
}

\author{
Raymond P Najjar (1) ${ }^{1,2}$ A V Rukmini, ${ }^{1}$ Maxwell T Finkelstein (1) , \\ Simon Nusinovici (ㄷ), 2,3 Baskaran Mani, ${ }^{2,4,5}$ Monisha Esther Nongpiur (ㅇ, , 2,4,5 \\ Shamira Perera, ${ }^{2,4}$ Rahat Husain, ${ }^{2,4}$ Tin Aung, ${ }^{2,4,5,6}$ Dan Milea (1) 1,2,7
}

\begin{abstract}
- Additional supplemental material is published online only. To view, please visit the journal online (http://dx.doi. org/10.1136/bjophthalmol2021-319938).
\end{abstract}

For numbered affiliations see end of article.

Correspondence to Dr Dan Milea, Singapore Eye Research Institute, Singapore; dan.milea@snec.com.sg

Preliminary findings were presented at the annual meeting of the Association of Research in Vision and Ophthalmology (ARVO) 2019, Vancouver, Canada (oral communication).

Received 23 June 2021 Accepted 6 November 2021

\section{Check for updates}

(C) Author(s) (or their employer(s)) 2021. Re-use permitted under CC BY-NC. No commercial re-use. See rights and permissions. Published by BMJ.

\section{To cite: Najjar RP,} Rukmini AV, Finkelstein MT, et al. Br J Ophthalmol Epub ahead of print: [please include Day Month Year]. doi:10.1136/

bjophthalmol-2021-319938

\section{ABSTRACT}

Background/aims Early detection and treatment of glaucoma can delay vision loss. In this study, we evaluate the performance of handheld chromatic pupillometry (HCP) for the objective and rapid detection of functional loss in glaucoma.

Methods In this clinic-based, prospective study, we enrolled 149 patients (median (IQR) years: 68.5 (13.6) years) with confirmed glaucoma and 173 healthy controls (55.2 (26.7) years). Changes in pupil size in response to $9 \mathrm{~s}$ of exponentially increasing blue (469 $\mathrm{nm}$ ) and red (640 nm) light-stimuli were assessed monocularly using a custom-built handheld pupillometer. Pupillometric features were extracted from individual traces and compared between groups. Features with the highest classification potential, selected using a gradient boosting machine technique, were incorporated into a generalised linear model for glaucoma classification. Receiver operating characteristic curve analyses (ROC) were used to compare the performance of HCP, optical coherence tomography (OCT) and Humphrey Visual Field (HVF).

Results Pupillary light responses were altered in glaucoma compared with controls. For glaucoma classification, HCP yielded an area under the ROC curve (AUC) of 0.94 (95\% Cl 0.91 to 0.96), a sensitivity of $87.9 \%$ and specificity of $88.4 \%$. The classification performance of HCP in early-moderate glaucoma (visual field mean deviation (VFMD) > -12 dB;AUC $=0.91$ (95\% $\mathrm{Cl} 0.87$ to 0.95$))$ was similar to HVF (AUC $=0.91)$ and reduced compared with $\mathrm{OCT}(\mathrm{AUC}=0.97 ; \mathrm{p}=0.01)$. For severe glaucoma (VFMD $\leq-12 \mathrm{~dB}), \mathrm{HCP}$ had an excellent classification performance (AUC $=0.98,95 \% \mathrm{Cl} 0.97$ to 1) that was similar to HVF and OCT.

Conclusion HCP allows for an accurate, objective and rapid detection of functional loss in glaucomatous eyes of different severities.

\section{INTRODUCTION}

Early detection and treatment of glaucoma can delay vision loss and consequently alleviate the health economic burden associated with the condition. ${ }^{12}$ However, glaucoma is often diagnosed at a late stage, especially in some low-income and middle-income countries, where up to $50 \%$ of patients are already blind in one eye at presentation. $^{34}$

The detection of glaucoma is challenging, in part because no standalone, affordable and accurate tool allows for it in an opportunistic or community setting. Existing methods, like optical coherence tomography (OCT) and standard automated perimetry (SAP) require expensive and highly calibrated apparatus. Furthermore, SAP is subjective, demanding in time and effort and does not always reflect the severity of structural decrements observed in early-stage glaucoma. ${ }^{56}$

Chromatic pupillometry, which measures the pupillary responses to light stimulations of different wavelengths, has become increasingly recognised as an effective method for evaluating retinal and optic nerve health. ${ }^{7}$ This technique investigates the integrity of retinal photoreceptors (ie, rods, cones and intrinsically photosensitive retinal ganglion cells (ipRGCs)) which contribute differentially to the pupillary light response (PLR). ${ }^{8-10}$ ipRGCs are a subset of RGCs that are intrinsically photosensitive ${ }^{8}$ and also integrate input from the outer retina, to mediate the PLR. ${ }^{10}$

Recent findings from our group and others suggest that ipRGC loss or dysfunction occurs in glaucoma, even at the earliest stages, resulting in abnormal pupillary responses to full-field or sectorial chromatic stimuli. ${ }^{11-13}$ In addition, multifocal stimuli targeting both subcortical (ie, ipRGC-driven responses) and adjacent corticopretectal (ie, originating from $\mathrm{M}$-cones and $\mathrm{L}$-cones through RGCs) chromatic pupillary responses, have been suggested to offer a higher sensitivity to glaucomatous functional alterations. ${ }^{14} 15$ Although chromatic pupillometry demonstrates high efficacy in the detection of functional abnormalities in glaucoma, the used lab-based devices are commonly bulky, expensive and unsuitable for everyday clinical use.

Using a custom-built handheld pupillometer, in this study, we evaluated the pupillary responses to exponentially increasing blue and red light stimulations in patients with different severities of glaucoma. Furthermore, we compared the performance of our handheld chromatic pupillometer (HCP) for glaucoma classification with established structural (ie, OCT) and functional (ie, SAP) investigation tools.

\section{MATERIALS AND METHODS \\ Participants}

Patients with unilateral or bilateral glaucoma and healthy controls were included in this clinic-based, cross-sectional study conducted between June 2017 and November 2018 (figure 1). In this prospective study, data collection was planned before pupillometric assessments and reference standard 


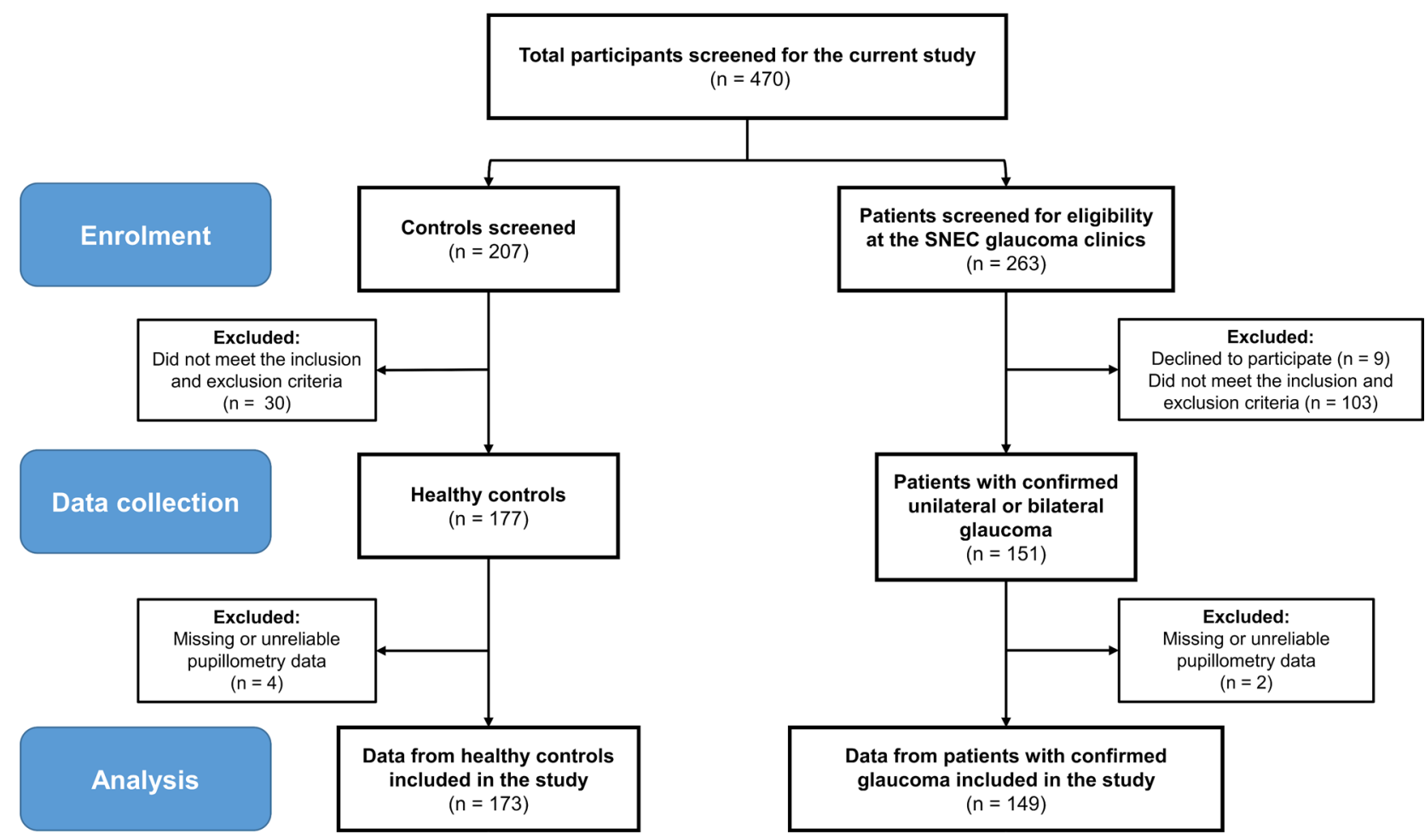

Figure 1 Diagram showing the flow of enrolment and exclusion of patients and data. A total of 470 participants including 207 controls and 263 patients were screened for this study. Out of the control group, 30 participants did not meet the study's inclusion and exclusion criteria and the pupillometry data collected from four control participants were unreliable $(n=2)$ or missing $(n=2)$. Out of 263 patients screened at the Singapore National Eye Centre (SNEC) glaucoma clinics, 9 declined to participate and 103 did not meet the study's inclusion and exclusion criteria. The pupillometry data collected from two glaucoma patients were missing or unreliable. This study included the data from 173 controls and 149 patients with confirmed unilateral or bilateral glaucoma.

confirmation. Glaucoma patients were recruited consecutively from the glaucoma clinics of glaucoma specialists AT and RH at the Singapore National Eye Centre (SNEC). Control participants were recruited from the general clinics of the hospital. All participants underwent a comprehensive ophthalmological evaluation (details in online supplemental methods 1 ). Controls were defined as subjects with no glaucomatous optic neuropathy (intraocular pressure less than $21 \mathrm{~mm} \mathrm{Hg}$, open iridocorneal angles (on gonioscopy) in all quadrants, healthy optic nerves and normal visual fields (VF)). Patients with clinically confirmed glaucoma were diagnosed by a fellowship trained specialist by the presence of glaucomatous optic neuropathy (loss of neuroretinal rim with a vertical cup-disc ratio of $>0.7$ and/or notching with nerve fibre layer defect attributable to glaucoma) with compatible VF defect. Based on Hodapp-ParishAnderson's criteria, patients were separated into early-moderatestage (VF mean deviation (VFMD) scores better than $-12 \mathrm{~dB}$ ) and severe-stage (VFMD scores of $-12 \mathrm{~dB}$ or worse) groups. ${ }^{16}$ This grouping was adopted to evaluate the performance of HCP in patients with less perceptible VF loss (early-moderate) and patients with severe glaucoma who are at highest risk for rapid worsening of the disease, lifetime blindness and deterioration of quality of life. ${ }^{17}$

Were also excluded patients with: (1) an unconfirmed glaucoma diagnosis or any associated ophthalmic conditions such as other causes of optic neuropathy, retinopathies and/or ocular motor disorders; (2) clinically significant pupillary abnormalities except for relative afferent pupillary defects (RAPD) in the glaucoma group; (3) clinically diagnosed psychiatric or neurological disorders, including dementia and (4) patients who had undergone intraocular surgery within the past 6 months, had severe ocular trauma or were using psychotropic or other medications known to affect the pupillary control pathways. Written informed consent was obtained from all participants. Research procedures adhered to ethical principles outlined in the Declaration of Helsinki. The full study protocol is available from the corresponding author on reasonable request.

\section{Handheld chromatic pupillometry}

Dynamic pupillary responses to chromatic stimulations in patients and controls were evaluated without dark adaptation using a custom-built handheld pupillometer in dimly-lit rooms $(<3$ lux $)$. A detailed description of the device and light calibration is provided in online supplemental methods 2, online supplemental figure 1. Experimenters were masked to the clinical information and final diagnosis of the participant during data acquisition.

Each participant underwent a 1 min pupillometric selfexamination that consisted of the following steps: $10 \mathrm{~s}$ of darkness (baseline pupil measurement); $9 \mathrm{~s}$ of exposure to exponentiallyincreasing blue light (11.7-14.4 log photons $/ \mathrm{cm}^{2} / \mathrm{s}$; peak wavelength $\left(\lambda_{\max }\right)=469 \mathrm{~nm}$, full width at half maximum $(\mathrm{FWHM})=33$ $\mathrm{nm}) ; 22 \mathrm{~s}$ of darkness to assess the dynamics of pupillary redilation to baseline; exposure to $9 \mathrm{~s}$ exponentially increasing red light stimulus $\left(11.9-14.3 \log\right.$ photons $/ \mathrm{cm}^{2} / \mathrm{s} ; \lambda=640 \mathrm{~nm}$, FWHM $=17 \mathrm{~nm}) ; 10 \mathrm{~s}$ of darkness to assess pupillary redilation (figure 2A). Light stimulations covered $50^{\circ}$ of the VF without considering reflections off the interior of the device. Participants were requested to fixate a central dim red zone ( $<0.1$ lux $)$ 
A

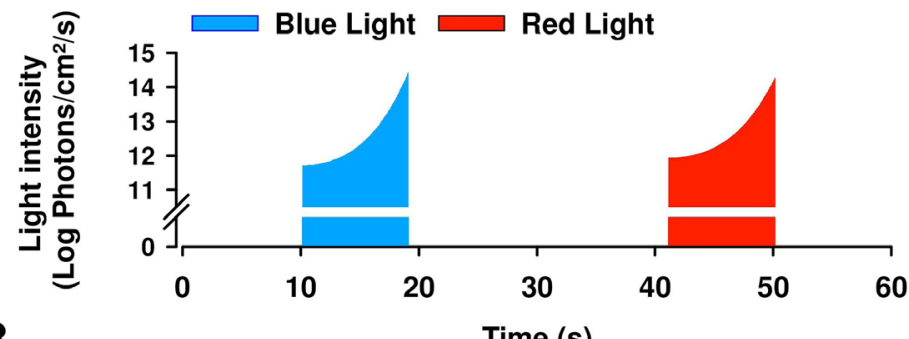

B

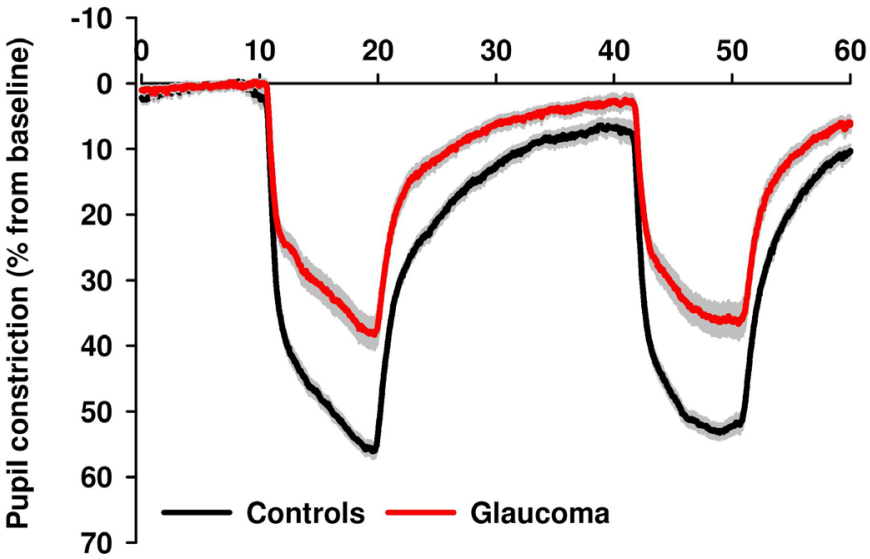

C
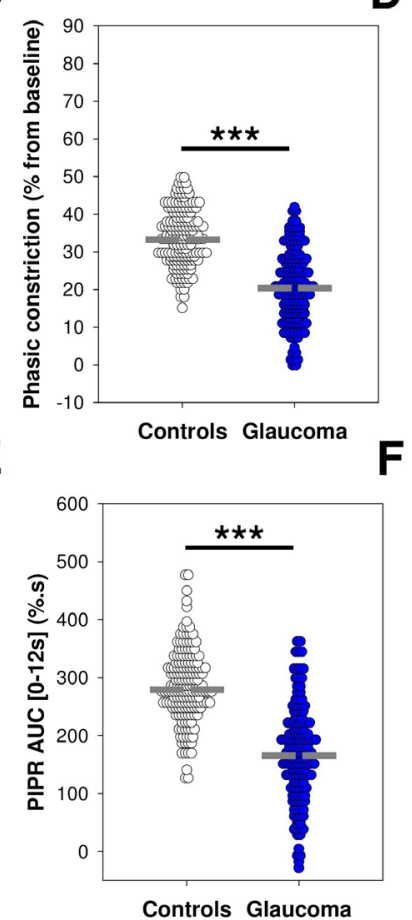

D
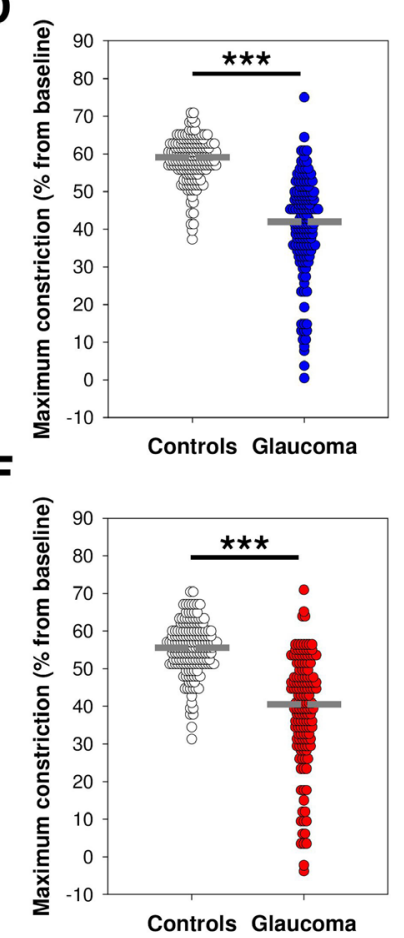

Figure 2 Average baseline-adjusted pupillary responses to the $1 \mathrm{~min}$ light paradigm in patients with glaucoma and controls. (A) After onset, light stimuli intensity increased exponentially from 11.7 to $14.4 \mathrm{log}$ photons $/ \mathrm{cm}^{2} / \mathrm{s}$ for blue light and from 11.9 to $14.3 \mathrm{log}$ photons $/ \mathrm{cm}^{2} / \mathrm{s}$ for red light. The duration of both blue and red light exposures was $9 \mathrm{~s}$. Blue light stimulus onset was preceded by $10 \mathrm{~s}$ of darkness to quantify baseline pupil size and followed by $22 \mathrm{~s}$ of darkness to assess pupillary redilation prior to red light onset. Red light was followed by $10 \mathrm{~s}$ of darkness to assess the pupil's redilation process. (B) Baseline-adjusted pupil constriction increased (pupil size decreased) rapidly at light onset and progressively as a function of the gradually increasing light exposure. In comparison to controls, patients with glaucoma exhibited significant alterations in the pupillary light responses. Data are plotted as average $\pm S E$. (C-F) Dot plots representing differences in main pupillometric features in response to blue $(C, D, E)$ and red (F) lights in patients with glaucoma compared with controls. The median of each group is represented as a dashed grey line. Statistical comparisons between groups were performed using a Mann-Whitney $\mathrm{U}$ test. ${ }^{* * *} \mathrm{p}<0.001$. AUC, area under the curve; PIPR, post-illumination pupillary response.

delivered using a red, green, blue (RGB) light-emitting diode (LED). Light was administered to the study eye, with the fellow eye covered by the participant's hand. In controls and patients with bilateral glaucoma and two eligible eyes, the study eye was selected randomly by coin toss.

\section{Data analysis and statistics}

Horizontal pupil radius measurements were individually processed using a semiautomated algorithm for blink artefact removal and expressed as a percentage change from baseline pupil size (figure 2B). This baseline correction accounted for any differences in baseline pupil size between participants and across groups. Sixteen principal pupillometric features were extracted from individual blink-free traces (online supplemental table 1).

Pupillometric features are presented as median (IQR) and were compared between controls and glaucoma groups using a Mann-Whitney U test. Demographics and ophthalmological examination outcomes are represented as median (IQR) and were compared between controls and glaucoma groups using a non-parametric Mann-Whitney $\mathrm{U}$ test or a $\chi^{2}$ test, and between controls and the different glaucoma severity groups using a Kruskal-Wallis One-Way analysis of variance on Ranks. Post hoc analysis was done pairwise using Dunn's method.

The relative contribution of demographic (age, gender and ethnicity), clinical (diabetes status) and pupillometric features in the training of the glaucoma classification model was determined using a gradient boosting machine (GBM) technique. A GBM is an ensemble of simple decision trees grown sequentially. The more a feature is associated with an improvement of the classification performance, the higher its relative contribution to the model. Top pupillometric contributing features (ie, excluding age, gender, ethnicity and diabetes status), corresponding to a cumulative relative contribution of at least $90 \%$ were used in a generalised linear model (GLM), a binary logistic regression that estimated the relationship between the outcome (no glaucoma/glaucoma) and continuous independent variables (pupillometric features). Adjusted ORs (adj. ORs) for pupillometric variables as well as their significance (Wald's test) were calculated. The classification performance of the GLM's fitted values (ie, glaucoma probability scores) was determined using a receiver operating characteristic curve (ROC) analysis. The area under the ROC curve (AUC), sensitivity, specificity, accuracy, positive and negative predictive values (PPV and NPV) were calculated at best statistical cut-off (highest Youden's J). Accuracy, PPV and NPV took into account a glaucoma prevalence of $3.54 \%$ in the general population (40-80 years old). ${ }^{18}$ Similar analytical approaches were used for OCT and Humphrey VF (HVF) features. The Akaike information criterion (AIC) was used as an indicator of the models' relative quality. Clinical diagnoses established on the same day as HCP by a fellowship trained specialist with 
Table 1 Demographics and clinical characteristics of the study eye in controls and patients with glaucoma

\begin{tabular}{|c|c|c|c|c|}
\hline & & Glaucoma & & \\
\hline & Controls & Early-moderate glaucoma & Severe glaucoma & All glaucoma \\
\hline $\mathrm{N}$ & 173 & 96 & 53 & 149 \\
\hline Age (years)ף & $55.2(26.7)$ & $68.5(14.2)^{* * *}$ & $68.3(14.3)^{* * *}$ & $68.5(13.6)^{* * *}$ \\
\hline Gender, no $(\% \text { male })^{*}$ & $80 / 173(46.2)$ & $56 / 96(58.3) \ddagger$ & $43 / 53(81.1)^{* * *}$ & $99 / 149(66.4)^{* * *}$ \\
\hline Ethnicity, no (\% Chinese)* & $157 / 173(90.8)$ & 91/96 (94.8)§ & $39 / 53(73.6)^{* *}$ & $130 / 149(87.2)$ \\
\hline Family history of glaucoma, no (\%)* & $5 / 173(2.9)$ & $23 / 96(24.0)^{* * *}$ & $9 / 53(17.0)^{* * *}$ & $32 / 149(21.5)^{* * *}$ \\
\hline Diabetes, no $(\% \text { with })^{*}$ & $15 / 173(8.7)$ & $25 / 96(26.0)^{* * *}$ & $14 / 53(26.4)^{* * *}$ & $39 / 149(26.2)^{* * *}$ \\
\hline Cataract, no (\% with)* & $46 / 173(26.6)$ & $55 / 96(57.3)^{* * *}$ & $33 / 53(62.3)^{* * *}$ & $88 / 149(59.1)^{* * *}$ \\
\hline PCIOL, no (\% with)* & $4 / 173(2.3)$ & $30 / 96(31.3)^{* * *}$ & $18 / 53(34.0)^{* * *}$ & $48 / 149(32.2)^{* * *}$ \\
\hline SE, median (IQR), DI & $-1.6(3.9)$ & $-1.1(4.9)$ & $-0.8(3.8)$ & $-0.9(4.4)$ \\
\hline BCVA, median (IQR), LogMarq & $0.08(0.2)$ & $0.1(0.2)^{* * *} \dagger$ & $0.3(0.3)^{* * *}$ & $0.2(0.2)^{* * *}$ \\
\hline VCDR, median (IQR)ף & $0.40(0.20)$ & $0.80(0.15)^{* * *} \dagger$ & $0.90(0.10)^{* * *}$ & $0.80(0.10)^{* * *}$ \\
\hline VFMD, median (IQR), dBף & $-1.0(2.1)$ & $-4.7(5.4)^{* * *} \S$ & $-18.6(9.6)^{* * *}$ & $-8.7(10.6)^{* * *}$ \\
\hline PSD, median (IQR), dBף & $1.6(0.7)$ & $4.5(5.3)^{* * *} \S$ & $11.1(4.5)^{* * *}$ & $7.7(7.4)^{* * *}$ \\
\hline VFI, median (IQR), \%ף & $99.0(2.3)$ & $90.0(14.0)^{* * *} \S$ & $51.5(32.8)^{* * *}$ & $79.5(30.5)^{* * *}$ \\
\hline Mean RNFL thickness, median (IQR), $\mu \mathrm{m}$ ๆ & $97.0(11.0)$ & $72.0(15.0)^{* * *} \ddagger$ & $58.0(10.5)^{* * *}$ & $68.0(18.8)^{* * *}$ \\
\hline RNFL symmetry, median (IQR), \%ף & $86.5(13.0)$ & $61.0(37.0)^{* * *} \dagger$ & $40.5(43.5)^{* * *}$ & $52.0(43.0)^{* * *}$ \\
\hline Rim area, median (IQR), $\mathrm{mm}^{2} \emptyset$ & $1.31(0.27)$ & $0.80(0.29)^{* * *}$ & $0.69(0.27)^{* * *}$ & $0.75(0.31)^{* * *}$ \\
\hline
\end{tabular}

A Kruskal-Wallis one way ANOVA on ranks was used to compare controls, early-moderate stage and severe stage glaucoma groups. A post-hoc pairwise comparison between the 3 groups was done using Dunn's method.

Difference between groups is indicated as ${ }^{* *} p<0.01 ;{ }^{* *} p<0.001$ for comparison with controls, and $+p<0.05 ; \ddagger p<0.01 ; \S p<0.001$ for pairwise comparison between earlymoderate and severe glaucoma.

If Statistics done using a Mann Whitney U-test between controls and all glaucoma group.

* Statistics done using a $\chi^{2}$ test.

ANOVA, analysis of variance; BCVA, best-corrected visual acuity; HVF, Humphrey visual field; LogMar, logarithm of minimum angle of resolution; PCIOL, posterior chamber intraocular lens; PSD, pattern SD; RNFL, retinal nerve fibre layer; SE, spherical equivalent; VCDR, vertical cup disk ratio; VFI, Visual Field Index; VFMD, visual field mean deviation.

access to participants' clinical records were used as reference standard. Pairwise comparison between the AUCs of HCP, OCT and HVF was performed using DeLong's method with Bonferroni correction. Machine learning and statistical procedures were performed using R V.3.6.3: A Language and Environment for Statistical Computing (R Core Team, Vienna, Austria). Figures were plotted using Sigmaplot V.14.0 (Systat Software, San Jose, California, USA). Intended sample size calculation is provided in online supplemental methods 3 .

\section{RESULTS}

\section{Participants}

A total of 470 participants were screened to take part in this study including 207 potential controls and 263 patients from the SNEC clinics. Of these, 142 participants did not meet the inclusion criteria of this study and the data of 6 participants (4 controls, 2 glaucoma) were excluded due to unreliable or missing pupillometry traces (figure 1). The data from 173 healthy controls (median age 55.2 (26.7) years, $46.2 \%$ males, $90.8 \%$ ethnic-Chinese) and 149 patients (68.5 (13.6) years, $66.4 \%$ males, $87.2 \%$ ethnic-Chinese) with bilateral or unilateral glaucoma were included in this study. The glaucoma group included 119 patients with primary open angle glaucoma (75 with high tension glaucoma and 44 with normal tension glaucoma), 27 patients with primary angle closure glaucoma and 3 patients with other glaucoma subtypes (1 pseudophakic, 1 pseudoexfoliation and 1 pigment dispersion glaucoma). Glaucoma patients were further divided into early-moderate and severe glaucoma groups. A detailed description of the demographics and clinical characteristics of all groups is provided in table 1. No adverse events were reported from any of the procedures used in the study.

\section{Pupillary responses to blue and red lights are altered in patients with glaucoma}

Baseline pupil size was reduced in patients with glaucoma compared with controls $(U=7302.0, \mathrm{p}<0.001)$ (online supplemental table 2). In both groups, the pupil, respectively, constricted in a phasic (fast) then gradual manner in response to the onset and exponential increase of the light stimuli (figure $2 \mathrm{~A}, \mathrm{~B}$ ). The average pupillary constriction curves were, however, markedly altered in the glaucoma group compared with controls (figure 2B). Multiple PLR features were different between groups. Principally, phasic constriction to blue $(U=3905 ; \mathrm{p}<0.001)$ and red $(U=4258$; $\mathrm{p}<0.001)$ lights, as well as maximum constriction to blue $(U=1912.5 ; \mathrm{p}<0.001)$ and $\mathrm{red}(U=3406.5 ; \mathrm{p}<0.001)$ lights were reduced in patients with glaucoma (figure $2 \mathrm{C}$, D and F; online supplemental table 2). Pupillary redilation was also affected by glaucoma as the area under the redilation curve after blue light offset (PIPR AUC 0-12 s) (figure 2E), the post-illumination pupillary responses $6 \mathrm{~s}$ after blue or red light offset (PIPR6s), as well as their difference (Net PIPR6s), were reduced in glaucomatous eyes (all, $\mathrm{p}<0.001$, online supplemental table 2).

\section{Relative feature contribution and classification performance of HCP for glaucoma}

The GBM analysis on 16 pupillometric features in addition to age, gender, ethnicity and diabetes status evaluated the importance of pupillometric and demographic variables in 
A

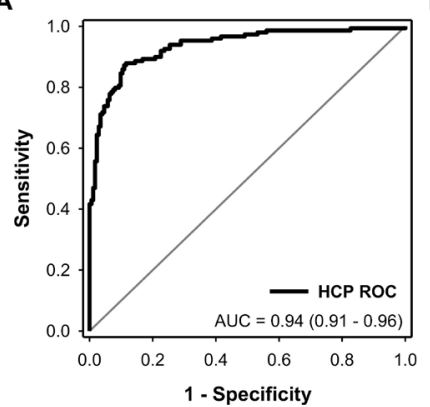

B

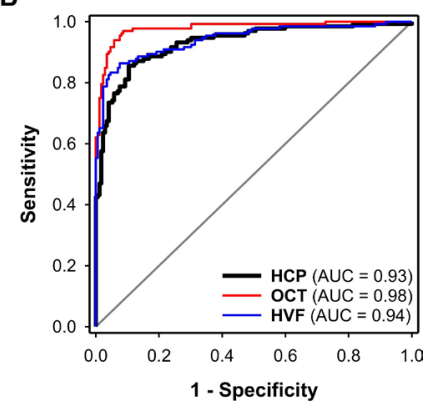

Figure 3 Receiver operating characteristic (ROC) curves reflecting the classification performance of HCP, OCT and HVF. (A) Receiver operating characteristic curve of HCP in 173 controls and 149 patients with glaucoma. The AUC of HCP was 0.94 ( $95 \% \mathrm{Cl} 0.91$ to 0.96$)$. (B) ROC curves of HCP, HVF and OCT in a similar population of 172 controls and 132 glaucoma patients. The AUC of HCP for the classification of glaucoma was $0.93(0.90-0.96)$ and was similar to that of HVF (0.94 (0.92-0.97); $p=0.56)$ but reduced compared with OCT (0.98 $(0.96-0.99) ; p=0.005)$. The AUC of HVF was significantly lower than OCT $(p=0.02)$. AUC, area under the curve; HCP, handheld chromatic pupillometry; HVF, Humphrey Visual Field; OCT, optical coherence tomography.

training the glaucoma classification model (online supplemental figure 2). The variable with the highest contribution to the classification model was maximum constriction to blue light (62.2\% relative contribution), followed by PIPR AUC 0-12 s and maximum constriction to red light with relative contributions of $7.3 \%$ and $6.3 \%$, respectively. Age and gender contributed $1.4 \%$ and $1.1 \%$ to the model, respectively, while the relative contributions of diabetes-status and ethnicity were negligible $(0 \%-0.1 \%)$ (online supplemental figure 2). Using top-contributing pupillometric features and excluding age, gender, ethnicity as well as diabetes status, we developed a GLM for glaucoma classification. The GLM $(\mathrm{AIC}=226.8)$ had an AUC of 0.94 (0.91-0.96), a sensitivity of $87.9 \%(81.6 \%-92.7 \%)$ and a specificity of $88.4 \%$ $(82.7 \%-92.8 \%$ ) (figure 3A). Significant variables in this model were maximum constriction to blue light $(\mathrm{p}=0.002)$ with an adj. OR of $0.21(0.08-0.56)$ per $10 \%$ increase in constriction and constriction latency to blue light $(\mathrm{p}=0.05)$ with an adj. OR of $1.41(1.00-1.98)$ per $100 \mathrm{~ms}$ increase in latency. To ensure that overfitting did not affect our results, we validated our model using a 10 -fold cross validation approach. The cross-validated AUC of the GLM was 0.93 (0.90-0.96). Detailed classification performance and crosstabulation results are shown in online supplemental table 3 . Interestingly, if maximum pupillary constriction to blue light was missing/removed from the model (eg, due to blinks or other artefacts), a GLM developed on the eight remaining pupillometric features, still maintains high classification performance (AUC $=0.93$, 95\% CI 0.90 to 0.96 ) with significant variables becoming PIPR AUC 0-12 s $(\mathrm{p}<0.001$, adj. OR of $0.80(0.71-0.91)$ per increase of $10 \% . s$ in the variable), maximum constriction to red light ( $\mathrm{p}<0.001$, adj. OR of $0.41(0.25-0.69)$ per increase of $10 \%$ in in the variable), constriction latency to blue light $(\mathrm{p}=0.04$, adj. OR of 1.40 (1.01 - 1.93) per increase of $100 \mathrm{~ms}$ in the variable), and constriction latency to red light $(\mathrm{p}=0.02$, adj. OR of 1.06 (1.01 - 1.11) per increase of $100 \mathrm{~ms}$ in the variable).

The exact statistical powers for the calculation of sensitivity and specificity of HCP were $96.5 \%$ and $97.2 \%$ for $\alpha=0.05$

Table 2 Comparison of the classification performance of HCP, OCT and HVF in a population of controls $(n=172)$ and patients with glaucoma

\begin{tabular}{|c|c|c|c|}
\hline & HCP & ОСТ & HVF \\
\hline \multicolumn{4}{|c|}{ All glaucoma $(n=132)$} \\
\hline AUC & 0.93 (0.90 to 0.96$)$ & $0.98(0.96 \text { to } 0.99)^{*}$ & $0.94(0.92$ to 0.97$) \dagger$ \\
\hline Sensitivity, \% & 85.6 (78.4 to 91.1$)$ & 97.0 (92.4 to 99.2$)$ & 86.4 (79.3 to 91.7$)$ \\
\hline Specificity, \% & 89.5 (84.0 to 93.7$)$ & 91.3 (86.0 to 95.0$)$ & $92.4(87.4$ to 95.9$)$ \\
\hline PPV, \% & 23.1 (16.2 to 31.9$)$ & 29.0 (20.1 to 39.9) & 29.5 (19.8 to 41.5$)$ \\
\hline NPV, \%‡ & 99.4 (99.1 to 99.6) & 99.9 (99.7 to 1) & 99.5 (99.2 to 99.7$)$ \\
\hline Accuracy, \%‡ & 89.4 (85.4 to 92.6$)$ & 91.5 (87.8 to 94.3$)$ & $92.2(88.6$ to 95.0$)$ \\
\hline \multicolumn{4}{|c|}{ Early-moderate glaucoma $(n=87)$} \\
\hline AUC & 0.91 (0.87 to 0.95$)$ & $0.97(0.95 \text { to } 0.99)^{*}$ & $0.91(0.87$ to 0.96$) \dagger$ \\
\hline Sensitivity, \% & 81.6 (71.9 to 89.1) & 95.4 (88.6 to 98.7$)$ & $79.3(69.3$ to 87.3$)$ \\
\hline Specificity, \% & 90.1 (84.6 to 94.1$)$ & 92.4 (87.4 to 95.9$)$ & $92.4(87.4$ to 95.9$)$ \\
\hline \multicolumn{4}{|c|}{ Severe glaucoma $(n=45)$} \\
\hline AUC & 0.98 (0.97 to 1$)$ & $1(1$ to 1$)$ & $1(1$ to 1$)$ \\
\hline Sensitivity, \% & 95.6 (84.9 to 99.5$)$ & $100(92.1$ to 100$)$ & 100 (92.1 to 100$)$ \\
\hline Specificity, \% & 94.2 (89.6 to 97.2 ) & 97.7 (94.2 to 99.4$)$ & $100(97.9$ to 100$)$ \\
\hline
\end{tabular}

Data are represented as average $(95 \% \mathrm{Cl})$. The statistical pairwise comparison of AUCs was performed using DeLong's method.

${ }^{*} \mathrm{p}<0.05$ between HCP and OCT, after Bonferroni correction. $t p<0.05$ between HVF and OCT, after Bonferroni correction.

$¥$ Values calculated at a disease prevalence of $3.54 \%$.

AUC, area under the curve; HCP, handheld chromatic pupillometry; HVF, Humphrey Visual Field; NPV, negative predictive value; $\mathrm{OCT}$, optical coherence tomography; PPV, positive predictive value.

at the disease prevalence of $46 \%$ observed in the final sample $(n=322)$.

\section{Comparison of performance characteristics between HCP, OCT and HVF}

Using a subgroup of patients and controls with no missing OCT and HVF variables (172 controls; 132 glaucoma) we adopted the same GBM approach and developed GLMs for glaucoma classification using top contributing features of OCT (by order of importance: mean RNFL thickness, rim area, VCDR, RNFL symmetry) and HVF (by order of importance: VF Index, VFMD and pattern SD). The AIC's of the GLMs were 218.8, 117.3 and 180.8 for HCP, OCT and HVF, respectively. The AUC of HCP for the detection of glaucoma was $0.93(0.90-0.96)$ and was similar to that of HVF $(0.94$ (0.92-0.97); $Z=-0.59 ; \mathrm{p}=0.56)$ but reduced compared with OCT (0.98 (0.96-0.99); $Z=-2.83 ; \mathrm{p}=0.005)$ (figure $3 \mathrm{~B}$, table 2). The AUC of HVF was significantly lower than OCT $(Z=2.42 ; \mathrm{p}=0.02)$ (figure $3 \mathrm{~B}$, table 2$)$.

Classification performance of HCP in a subgroup of glaucoma patients and controls matched for age, diabetes and cataract surgery statuses

To further consolidate our findings, we performed a subgroup analysis after matching controls and glaucoma groups for age (controls: 66.3 (6.0) years; glaucoma: 68.0 (5.3) years; $U=1940.5, \mathrm{p}=0.61)$, diabetes $\left(\chi^{2}=1.36, \mathrm{p}=0.25\right)$ and cataract surgery $\left(\chi^{2}=0.43, p=0.51\right)$ statuses. In this sample of 64 controls and 64 glaucoma patients, using a new GLM, the AUC of HCP remained high (0.93 (0.88-0.97)), while sensitivity was $92.2 \%$ and specificity $81.3 \%$. The classification performance of HCP was not significantly different from that of HVF (AUC $=0.92,95 \%$ CI 0.86 to 0.97$)$ and OCT $(\mathrm{AUC}=0.97,95 \% \mathrm{CI} 0.95$ to 1$)$. 


\section{Classification performance of HCP across glaucoma severity groups}

Alterations in the pupillary constriction profiles were dependent on the severity of glaucoma (online supplemental figure $3 \mathrm{~A})$. For example, maximum constriction to blue light gradually decreased with the severity of the disease $(H=182.4, \mathrm{p}<0.001$; online supplemental figure $3 \mathrm{~B}$ ). Using specific GLMs developed using HCP features in controls and different sub-groups of glaucoma severity, the AUC of HCP for the detection of earlymoderate stage glaucoma was $0.91(0.87-0.95)$ and was similar to HVF (0.91 (0.87-0.96); $Z=-0.13 ; \mathrm{p}=0.90)$ and reduced compared with OCT $(0.97(0.95-0.99) ; Z=-2.56 ; \mathrm{p}=0.01)$ (table 2). Conversely, the performance of HCP for the detection of severe glaucoma (AUC $=0.98,95 \%$ CI 0.97 to 1 ) was similar to OCT and HVF (table 2).

\section{DISCUSSION}

In this study, we demonstrate that pupillometric dysfunctions occur in glaucoma and can be revealed with a custom-built handheld pupillometer, delivering tailored, fast, chromatic light paradigms. Furthermore, a model including predominant pupillometric features of the disease can be used to detect functional glaucomatous loss with high accuracy.

The reactivity of the pupil to light is dependent on the integrity of retinal photoreceptors and their neural circuitry. ${ }^{19} \mathrm{We}$ previously demonstrated that ramping-up chromatic light stimulations demonstrate reduced PLRs in different severities of glaucoma. ${ }^{11}$ The method was subsequently shown not to be affected by mild and moderate cataracts or refractive errors, ${ }^{20} 21$ with the potential of detecting subtle functional deficits in eyes with earlystage glaucoma. ${ }^{13}$ Using a handheld pupillometer that delivers a similar, yet shortened and refined light paradigm, our study confirms altered PLRs in patients with glaucoma and underlines the effectiveness of direct, monocular, HCP for the detection of functional loss in the disease.

Our results show alterations in the ipRGC-driven pupillary response which are in agreement with electrophysiological reports in patients with glaucoma, ${ }^{22}$ and findings in rodent models of chronic ocular hypertension. ${ }^{23}$ Interestingly, among the various pupillometric features altered in glaucomatous eyes, maximum constriction to blue light $\left(14.4 \log\right.$ photons $\left./ \mathrm{cm}^{2} / \mathrm{s}\right)$, and not the PIPR or Net PIPR, ${ }^{2}$ showed the highest relative contribution to the classification performance of the GBM model. These findings suggest that the pupillometric hallmarks of ipRGC integrity are also dependent on the pattern of a light stimulation. In principle, our gradually increasing light stimulations evaluated the integrated extrinsic and intrinsic ipRGCs response, ${ }^{11}{ }^{13}$ but would likely yield maximum intrinsic (ie, melanopsin) stimulation and contribution at peak blue light intensity. ${ }^{8}$ In addition to RGC damage, electrophysiological and histological studies have also suggested alterations in outer retinal photoreception in glaucoma. ${ }^{24}$ Here, alterations in the latency and phasic pupillary constriction amplitudes, in addition to changes in the early redilation phases of the PIPR $(<1.7 \mathrm{~s})$ suggested to involve rods, ${ }^{26}$ are in agreement with the aforementioned literature and suggest outer retinal dysfunction in glaucoma.

Findings from previous studies focusing on the RAPD were only moderately encouraging with AUCs ranging between 0.74 and $0.85 .^{27-30}$ This was probably due to the reduced classification performance of RAPD features in symmetric glaucoma, ${ }^{27}$ leading to the categorisation of pupillometers as devices with limited value for glaucoma detection. The performance characteristics of direct or consensual pupillometry for the detection of functional loss in glaucoma were also evaluated by multiple groups. ${ }^{12} 14{ }^{31-34}$ Using the direct pupillary response to shapelike stimulations, ${ }^{34}$ or consensual PIPR to quadrant stimulations, ${ }^{12}$ pupillometry yielded high classification accuracy (AUCs $>0.90$ ) for glaucoma. Conversely, chromatic multifocal infrared pupillometry only showed fair to good diagnostic accuracy with AUCs ranging from 0.71 to 0.82 for the detection of mild and moderate to severe glaucoma, respectively. ${ }^{14}$ While these original approaches used differential spatial and multifocal stimulation of the retina, the studies had small sample sizes, and relied on devices that required good fixation and unfailing cooperation from patients. Using the RAPDx system (Konan Medical, California, USA), a 7 min stimulation sequence and an elaborate analytical strategy, Chang et al showed that the performance was lowest $(\mathrm{AUC}=0.78)$ when comparing results between eyes and increased (AUC $=0.87$ ) in a model including asymmetry in pupillometric outcomes and age. ${ }^{35}$ With a similar analytical approach applied only to pupillometric features, in this proof-of-concept study, we show that a low-cost handheld pupillometer delivering short-duration light stimulations, requiring minimal fixation aptitudes and no dark-adaptation, can highlight functional loss in eyes with glaucoma. Similar fast pupillometry-based approaches are also showing promise for the fast detection of functional loss in other ocular conditions. ${ }^{36}$

From an analytical standpoint, although only two pupillometric features were significant among nine to predict glaucoma using a GLM, keeping all the nine features would have practical interest. For instance, if a patient blinks at peak blue light intensity, the feature with the highest relative contribution, that is, maximum constriction to blue light, may become unreliable. Using the eight remaining pupillometric features (online supplemental figure 2) in a new GLM-potentially embedded in an analysis software-HCP can still detect functional loss in glaucoma with high accuracy (AUC of $0.93(0.90-0.96)$ instead of $0.94(0.91-0.96)$. This approach could improve the reliability of a testing procedure, reduce administration time, and prevent the repetition of a test if artefacts such as blinks were to mask on one or more pupillometric features.

Glaucoma is a treatable disease, nonetheless the efficacy of a treatment is dependent on the severity of the condition at diagnosis. ${ }^{37}$ In the USA, more than US\$2500/patient/year is spent on advanced glaucoma, compared with US\$623 on early glaucoma. ${ }^{38}$ Nevertheless, the prevalence of undiagnosed and untreated glaucoma remains high in developed countries and alarmingly so in the developing world, reaching magnitudes over $95 \%$ in the rural parts of some countries. ${ }^{39}$ Major advances in telemedicine and artificial intelligence, may yet reduce the cost of accurate glaucoma detection. Considering its high accuracy (89.4\%) and NPV (>99\%), and its promise to detect other sight threatening conditions, ${ }^{7}$ HCP's aptitudes for glaucoma detection should be investigated further in a dedicated screening setting (eg, community, general practitioner's clinic). Furthermore, at a fraction of the cost (ie, prototype excluding iOS system and software development costs at $\sim$ US\$450), its performance is similar to that of OCT and HVF in detecting severe glaucoma. Thus, HCP has the potential to alleviate burden of blindness on first presentation to glaucoma clinics in developing countries. ${ }^{3}$ Future studies should evaluate the potential of pupillometry in general and HCP in particular for the tracking of glaucoma progression.

Our study has some limitations, inherent to the novelty of this proof-of-concept investigation. First, it was performed in a clinical setting where the prevalence of glaucoma is higher than the community. Without a prospective community-based study, we are therefore unable to infer whether HCP would yield similar diagnostic performance in a non-clinical setting or a less selected, multiethnic population with inherently different demographic and clinical 
characteristics. Second, given our intent to evaluate the performance of HCP in a busy and time-sensitive clinical setting, we did not darkadapt or pharmacologically dilate the pupils of our participants. Therefore, while our method is likely to underestimate the absolute magnitude of PLRs in participants, our findings are all the more applicable in clinical, time-constrained settings. Third, abnormal PLRs may also be present in other ocular or even neurological conditions. ${ }^{7041}$ Although that may decrease the specificity of HCP for glaucoma, the ability to uncover multiple ocular diseases is valuable for screening strategies. ${ }^{3}$

In conclusion, our proof-of-concept study highlights the feasibility and high accuracy of chromatic pupillometry delivered using a handheld device for the objective detection of functional loss in glaucoma in a clinical setting. Further community-based or opportunistic screening studies, as well as cost-effectiveness analyses, are necessary to confirm whether HCP is a useful tool for glaucoma screening.

\author{
Author affiliations \\ 1Visual Neurosciences Research Group, Singapore Eye Research Institute, Singapore \\ ${ }^{2}$ Ophthalmology and Visual Sciences Academic Clinical Programme, Duke-NUS \\ Medical School, Singapore \\ ${ }^{3}$ Ocular Epidemiology Research Group, Singapore Eye Research Institute, Singapore \\ ${ }^{4}$ Glaucoma Department, Singapore National Eye Centre, Singapore \\ ${ }^{5}$ Glaucoma Research Group, Singapore Eye Research Institute, Singapore \\ ${ }^{6}$ Department of Ophthalmology, Yong Loo Lin School of Medicine, National University \\ of Singapore, Singapore \\ ${ }^{7}$ Neuro-Ophthalmology Department, Singapore National Eye Center, Singapore
}

\section{Twitter Raymond P Najjar @Ray_Najjar and Dan Milea @DanMilea3}

Acknowledgements The authors thank the clinical research coordinators of the Glaucoma Department at the Singapore Eye Research Institute for their efforts in recruiting patients.

Contributors Concept and design: RPN, BM and DM. Data acquisition and research execution: RPN, AVR, MTF, BM, MEN, SP, RH, TA and DM. Analysis and interpretation: RPN, MTF, SN, MEN, TA and DM. Manuscript preparation: RPN, AVR, MTF, SN, SP, RH, TA and DM. Guarantors of the study: RPN and DM.

Funding This work was supported by the National Medical Research Council, Singapore (NMRC/CIRG/1401/2014) and the National Health Innovation Centre Singapore (NHIC-I2D-1708181) to DM.

Disclaimer The funding organisations organisations had no role in the design and conduct of the research.

Competing interests DM has a patent application based on the pupillometry protocol used in the present study (PCT/SG2015/050494): A method and system for monitoring and/or assessing pupillary responses. RPN, TA and DM have a patent application based on the handheld pupillometer used in this study (PCT/ SG2018/050204): Handheld ophthalmic and neurological screening device. The rest of the authors have no conflicts of interest to disclose.

Patient consent for publication Consent obtained directly from patient(s)

Ethics approval The study was approved by the SingHealth Centralised Institutional Review Board (CIRB (2018/3233)).

Provenance and peer review Not commissioned; externally peer reviewed.

Data availability statement The de-identified datasets used in this study can be made available from the authors upon reasonable request. The full study protocol is available from the corresponding author on reasonable request.

Supplemental material This content has been supplied by the author(s). It has not been vetted by BMJ Publishing Group Limited (BMJ) and may not have been peer-reviewed. Any opinions or recommendations discussed are solely those of the author(s) and are not endorsed by BMJ. BMJ disclaims all liability and responsibility arising from any reliance placed on the content. Where the content includes any translated material, BMJ does not warrant the accuracy and reliability of the translations (including but not limited to local regulations, clinical guidelines, terminology, drug names and drug dosages), and is not responsible for any error and/or omissions arising from translation and adaptation or otherwise.

Open access This is an open access article distributed in accordance with the Creative Commons Attribution Non Commercial (CC BY-NC 4.0) license, which permits others to distribute, remix, adapt, build upon this work non-commercially, and license their derivative works on different terms, provided the original work is properly cited, appropriate credit is given, any changes made indicated, and the use is non-commercial. See: http://creativecommons.org/licenses/by-nc/4.0/.

\section{ORCID iDs}

Raymond P Najjar http://orcid.org/0000-0002-3770-2300

Maxwell T Finkelstein http://orcid.org/0000-0002-9039-4546

Simon Nusinovici http://orcid.org/0000-0002-9599-5818

Monisha Esther Nongpiur http://orcid.org/0000-0003-2372-8523

Dan Milea http://orcid.org/000-0002-6388-8639

\section{REFERENCES}

1 Bramley T, Peeples P, Walt JG, et al. Impact of vision loss on costs and outcomes in medicare beneficiaries with glaucoma. Arch Ophthalmol 2008;126:849-56.

2 Chua J, Baskaran M, Ong PG, et al. Prevalence, risk factors, and visual features of undiagnosed glaucoma: the Singapore epidemiology of eye diseases study. JAMA Ophthalmol 2015;133:938-46.

3 Jones PR, Philippin H, Makupa WU, et al. Severity of visual field loss at first presentation to glaucoma clinics in England and Tanzania. Ophthalmic Epidemiol 2020;27:10-18.

4 Cook C. Glaucoma in Africa: size of the problem and possible solutions. J Glaucoma 2009;18:124-8

5 Leung CKS, Liu S, Weinreb RN, et al. Evaluation of retinal nerve fiber layer progression in glaucoma a prospective analysis with neuroretinal rim and visual field progression. Ophthalmology 2011;118:1551-7.

6 Glen FC, Baker H, Crabb DP. A qualitative investigation into patients' views on visual field testing for glaucoma monitoring. BMJ Open 2014;4:e003996.

7 Rukmini AV, Milea D, Gooley JJ. Chromatic pupillometry methods for assessing photoreceptor health in retinal and optic nerve diseases. Front Neurol 2019;10:76.

$8 \mathrm{McDougal}$ DH, Gamlin PD. The influence of intrinsically-photosensitive retinal ganglion cells on the spectral sensitivity and response dynamics of the human pupillary light reflex. Vision Res 2010;50:72-87.

9 Kardon R, Anderson SC, Damariian TG, et al. Chromatic pupil responses: preferentia activation of the melanopsin-mediated versus outer photoreceptor-mediated pupil light reflex. Ophthalmology 2009;116:1564-73.

10 Gooley JJ, Ho Mien I, St Hilaire MA, et al. Melanopsin and rod-cone photoreceptors play different roles in mediating pupillary light responses during exposure to continuous light in humans. J Neurosci 2012;32:14242-53.

11 Rukmini AV, Milea D, Baskaran M, et al. Pupillary responses to high-irradiance blue light correlate with glaucoma severity. Ophthalmology 2015;122:1777-85.

12 Adhikari P, Zele AJ, Thomas R, et al. Quadrant field pupillometry detects melanopsin dysfunction in glaucoma suspects and early glaucoma. Sci Rep 2016;6:33373.

13 Najjar RP, Sharma S, Atalay E, et al. Pupillary responses to Full-Field chromatic stimuli are reduced in patients with early-stage primary open-angle glaucoma. Ophthalmology 2018;125:1362-71.

14 Carle CF, James AC, Kolic M, et al. Blue multifocal Pupillographic objective perimetry in glaucoma. Invest Ophthalmol Vis Sci 2015;56:6394-403.

15 Carle $C F$, James AC, Kolic M, et al. Luminance and colour variant pupil perimetry in glaucoma. Clin Exp Ophthalmol 2014;42:815-24

16 Hodapp E, Parrish RK, Anderson DR. Clinical decisions in glaucoma. St. Louis: The CV Mosby Company, 1993.

17 Kastner A, King AJ. Advanced glaucoma at diagnosis: current perspectives. Eye 2020;34:116-28

18 Tham Y-C, Li X, Wong TY, et al. Global prevalence of glaucoma and projections of glaucoma burden through 2040: a systematic review and meta-analysis. Ophthalmology 2014;121:2081-90.

19 Gamlin PDR, McDougal DH, Pokorny J, et al. Human and macaque pupil responses driven by melanopsin-containing retinal ganglion cells. Vision Res 2007;47:946-54

20 Rukmini AV, Milea D, Aung T, et al. Pupillary responses to short-wavelength light are preserved in aging. Sci Rep 2017;7:43832.

21 Rukmini AV, Chew MC, Finkelstein MT, et al. Effects of low and moderate refractive errors on chromatic pupillometry. Sci Rep 2019;9:4945.

22 Kuze M, Morita T, Fukuda Y, et al. Electrophysiological responses from intrinsically photosensitive retinal ganglion cells are diminished in glaucoma patients. J Optom 2017:10:226-32.

23 Wang H-zhou, Lu Q-jun, Wang N-li, Wang H, Lu Q, et al. Loss of melanopsincontaining retinal ganglion cells in a rat glaucoma model. Chin Med $\lrcorner$ 2008; 121:1015-9.

24 Fazio DT, Heckenlively JR, Martin DA, et al. The electroretinogram in advanced openangle glaucoma. Doc Ophthalmol 1986;63:45-54.

25 Nork TM, Ver Hoeve JN, Poulsen GL, et al. Swelling and loss of photoreceptors in chronic human and experimental glaucomas. Arch Ophthalmol 2000;118:235-45.

26 Adhikari P, Feigl B, Zele AJ. Rhodopsin and melanopsin contributions to the early Redilation phase of the post-illumination pupil response (PIPR). PLoS One 2016;11:e0161175

27 Rao HL, Kadambi SV, Mehta P, et al. Diagnostic ability of automated Pupillography in glaucoma. Curr Eye Res 2017;42:743-7 
28 Tatham AJ, Meira-Freitas D, Weinreb RN, et al. Detecting glaucoma using automated pupillography. Ophthalmology 2014;121:1185-93.

29 Chang DS, Xu L, Boland MV, et al. Accuracy of pupil assessment for the detection of glaucoma: a systematic review and meta-analysis. Ophthalmology 2013; 120:2217-25.

30 Kalaboukhova L, Fridhammar V, Lindblom B. Relative afferent pupillary defect in glaucoma: a pupillometric study. Acta Ophthalmol Scand 2007;85:519-25.

31 Feigl B, Mattes D, Thomas R, et al. Intrinsically photosensitive (melanopsin) retinal ganglion cell function in glaucoma. Invest Ophthalmol Vis Sci 2011;52:4362-7.

32 Kankipati L, Girkin CA, Gamlin PD. The post-illumination pupil response is reduced in glaucoma patients. Invest Ophthalmol Vis Sci 2011;52:2287-92.

33 Kelbsch C, Maeda F, Strasser T, et al. Pupillary responses driven by ipRGCs and classical photoreceptors are impaired in glaucoma. Graefes Arch Clin Exp Ophthalmol 2016;254:1361-70.

34 Wride N, Habib M, Morris K, et al. Clinical evaluation of a rapid, pupil-based assessment of retinal damage associated with glaucoma. Clin Ophthalmol 2009;3:123-8.
35 Chang DS, Arora KS, Boland MV, et al. Development and validation of an associative model for the detection of glaucoma using pupillography. Am J Ophthalmol 2013; 156:1285-96.

36 Maddess T, Rai BB, Carle C. Diagnostic power of rapid ETDRS-grid matched objective perimetry in early- to late-stage AMD. Invest Ophthalmol Vis Sci 2021;62:316.

37 Grant WM, Burke JF. Why do some people go blind from glaucoma? Ophthalmology 1982;89:991-8.

38 Lee PP, Walt JG, Doyle JJ, et al. A multicenter, retrospective pilot study of resource use and costs associated with severity of disease in glaucoma. Arch Ophthalmol 2006;124:12-19.

39 Thapa SS, Paudyal I, Khanal S, et al. A population-based survey of the prevalence and types of glaucoma in Nepal: the Bhaktapur glaucoma study. Ophthalmology 2012;119:759-64

40 Chougule PS, Najjar RP, Finkelstein MT, et al. Light-Induced pupillary responses in Alzheimer's disease. Front Neurol 2019:10:360.

41 Ali EN, Carle CF, Lueck CJ, et al. Assessing migraine patients with multifocal pupillographic objective perimetry. BMC Neurol 2021;21:211. 